\title{
Intussusception After Roux-en-Y Gastric Bypass in a Pregnant Patient
}

\author{
Bandar Idrees Ali a, b, Mohammed Al-Jabalia, Abdullah Al-Masoud ${ }^{\mathrm{a}}$, \\ Abdullah Al-Zahrania
}

\begin{abstract}
Intussusception is a rare complication following Roux-en-Y gastric bypass surgery, and can be present with various complaints making it difficult to diagnose and even more challenging in the pregnant patients. We describe a case of a 32-year-old woman at 24 weeks of gestation that presented with a progressive onset of central abdominal pain over 3 days. On exploration, an intussusception with necrotic bowel was found, so resection of the affected bowel and reconstruction were made. With the increasing number of bariatric procedures being performed in obese women of childbearing age, practitioners should be aware of this serious complication.
\end{abstract}

Keywords: Intussusception; Roux-en-Y gastric bypass; Pregnancy; Bariatric; Obesity

\section{Introduction}

Roux-en-Y gastric bypass (RYGB), developed and refined over the last 40 years, is one of the most common weight loss procedures performed worldwide [1]. The adoption of the laparoscopic approach improved the procedure's morbidity and facilitated widespread acceptance and implementation [1]. RYGB achieves significant and sustained weight loss with an acceptably low complication profile. Among the most worrisome of late-presenting complications is a bowel obstruction. Adhesions or internal hernias are mostly to be blamed, though rarely obstruction can be the result of intussusceptions $[2,3]$. It occurs usually near the jejunojejunostomy (JJ) and all three limbs of the gastric bypass can be affected. Dysmotility disorders are the most accepted etiology for intussusception after laparoscopic RYGB. Simple reduction +/- plication and resection with reconstruction of the JJ are two feasible therapeu-

Manuscript accepted for publication May 18, 2016

aDivision of Gastrointestinal Surgery, Department of Surgery, Prince Sultan Military Medical City, Riyadh, Saudi Arabia

${ }^{b}$ Corresponding Author: Bandar Idrees Ali, Division of Gastrointestinal Surgery, Department of Surgery, Prince Sultan Military Medical City, Riyadh, Saudi Arabia.Email: biaa1003@yahoo.com

doi: http://dx.doi.org/10.14740/jmc2534w tic options. Fertility and libido increase in patients following bariatric surgery, and pregnancy in the following years after surgery is not unusual [4]. Few cases in the literature reported bowel obstruction in pregnant patients after RYGB $[5,6]$. In these cases, the median time to presentation after gastric bypass surgery was 36 months from the surgery and during their second trimester of pregnancy. Reported cases ended up by uneventful spontaneous delivery, intra-operative combined fetal delivery via emergency cesarean section or postoperative mortality for a fetus, mother or both.

\section{Case Report}

A gravida 4, para 3 of the 32-year-old woman at 32 weeks gestation developed progressive onset of a short period of epigastric abdominal pain and vomiting. Past history included laparoscopic RYGB for morbid obesity 5 years earlier. She had since lost $80 \mathrm{~kg}$. The patient appeared non-toxic, and the abdominal exam revealed a soft, gravid abdomen with mild tenderness in the epigastrium and peritoneal signs. External fetal monitoring showed no distress, and a sonogram confirmed viable fetus up to date with adequate amniotic fluid. The vaginal exam revealed closed cervix.

Laboratory studies were normal including a full blood cell count, as well as urea, coagulation profile, liver function test, and chemistry. While in the general floor, the patient's pain intensified and she had multiple episodes of coffee-ground vomiting. Upper GI endoscopy showed multiple ulcers over the stapler line with no active bleeding with recommendation to start omeprazole (IV) as a prophylactic measure. As patient showed no clinical improvement over 3 days, decision for computed tomography (CT) abdomen was taken for highly suspicious for mesenteric venous occlusion. The $\mathrm{CT}$ finding suggested a closed loop bowel obstruction with possible volvulus (Fig. 1). The patient was administered tocolytic (IV), antibiotics, vigorous IVF and taken to the operating room for exploratory laparotomy.

At exploration, the Roux limb was dilated with severe venous congestion (Fig. 2). The obstruction was being caused by a retrograde intussusception of the JJ into the common channel exactly $15 \mathrm{~cm}$ in the common limb. The enrolled segment " $20 \mathrm{~cm}$ was resected" and bowel continuity was re-established using linear stapler between the JJ and common channel. The procedure was completed including the closure of the mesen- 


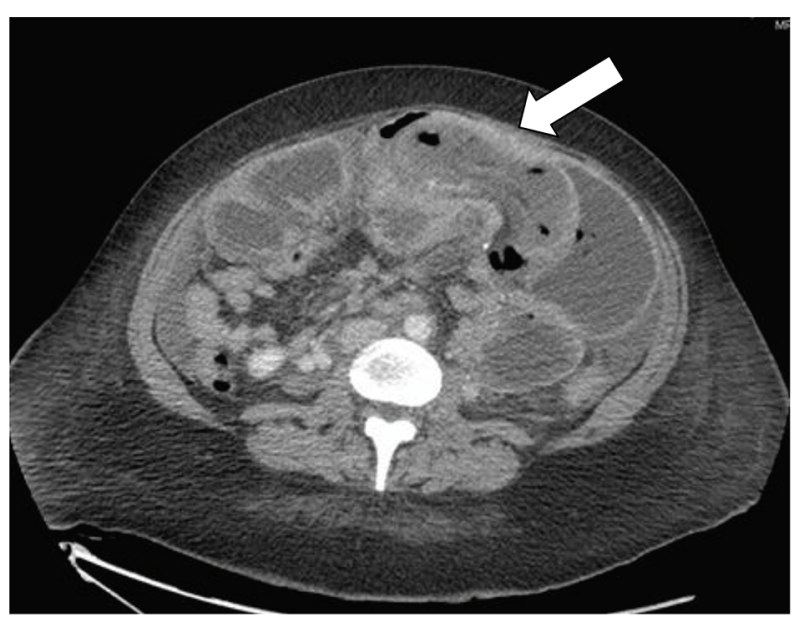

Figure 1. Axial (cross-section) view of CT abdomen shows the jejunojejunal intussusception of the alimentary loop with the typical (target sign) marked by the arrow.

\section{teric defect.}

The patient had an uneventful postoperative course. Pathology of the resected small bowel revealed ischemic enteritis with mucosal and patchy transmural necrosis.

\section{Discussion}

Intussusception is a rare condition in the adult population, accounting for only $1-5 \%$ of cases of bowel obstruction $[7,8]$. In more than $90 \%$ of adult cases, a lead point can be identified, usually lymphoid hyperplasia or malignancy $[9,10]$. There are 71 cases of intussusceptions after RYGB reported in the literature, and we are not sure for the pregnant cases as in our patient [3].

The mechanism of intussusception is not clearly understood. In cases where there is a pathological lead point lesion, the trailing contiguous bowel (the intussusceptum) telescopes into adjacent proximal or distal bowel (the intussuscipiens). This traps and kinks the mesentery of the intussusceptum, causing vascular congestion, bowel wall edema, pain, and eventually ischemic necrosis. The junction between free and fixed portions of the bowel is an area particularly prone to intussusception. Like in normal anatomy, the ileum can intussuscept into the right colon. With surgical rerouting and anastomosis, other segments of bowel can become fixed in relation to the adjacent intestine. The extreme weight loss in RYGB patients leaves a floppy mobile mesentery that gives the unfixed bowel even greater mobility to intussuscept, and staple lines in these patients may function as lead points. RYGB can also predispose to intussusception with dysmotility of Roux limb peristalsis as documented in an actual case as well as in a dog model.

The majority of people with intussusception present with pain, nausea, and vomiting. Peritonitis is not seen unless necrosis or perforation has occurred. A palpable mass is rarely appreciated, even in the absence of a gravid uterus and displaced bowel [3]. In patients who have had weight loss surgery, this obstructive-type presentation should "set off alarms", and full

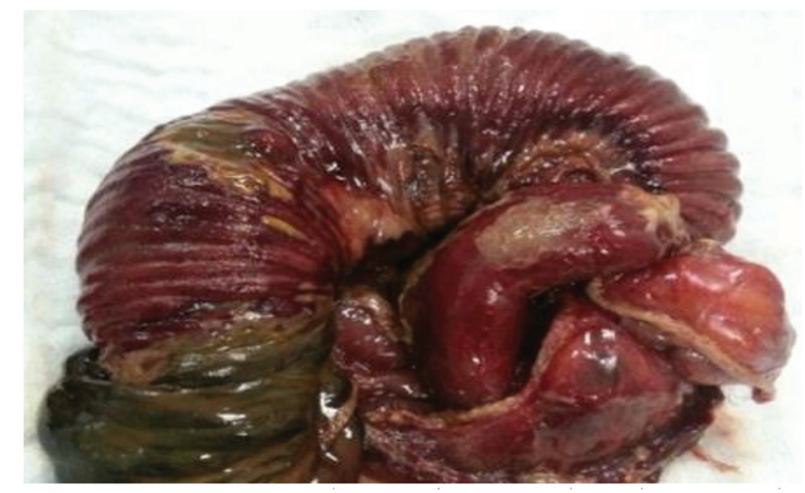

Figure 2. Gross specimen of resected segment of bowel shows hyperemic thickened and necrotic patches.

workup with appropriate imaging to exclude operative pathology should be undertaken. CT scan with oral and intravenous contrast is the most frequent study performed in this presentation, though the use of ultrasound or magnetic resonance imaging (MRI) would be an option, especially in the first trimester of pregnancy. Regardless of the mode of imaging, the lead point is frequently missed as it is often small and hidden within the density of the intussusceptum mass. Several studies show CT sensitivity to be at $66-78 \%[2,3]$.

Ultrasound is a fast study that can have high accuracy, but only in experienced hands. MRI can also capably demonstrate intussusception due to the high contrast resolution between an intraluminal fluid and the bowel wall, though the study is often cumbersome to obtain, limiting its clinical usefulness in the acute setting [8]. In the second or third trimester of pregnancy, a CT scan represents little risk to the fetus and is a safe option for the rapid diagnosis of a potentially life-threatening condition to both the mother and fetus. This is especially true in the face of other equivocal study results, where definitive CT findings would influence the management decisions [10].

In the majority of cases of intussusception, definitive diagnosis is only made at the time of operation $[7,9,10]$. Intraoperative reduction and/or resection of the involved bowel are mandatory to exclude irreversible ischemia and necrosis. Prognosis is variable, with mortality rates for intestinal intussusception reported between $1 \%$ and $16 \%$ [10]. A mortality rate of $50 \%$ was reported when surgical intervention was delayed by more than $48 \mathrm{~h}$ after the onset of symptoms. This underlines the importance of working up these patients who present with obstructive symptoms following RYGB and proceeding early to definitive diagnosis and management, ideally with diagnostic laparoscopy where appropriate. The presentation may complicate pregnancy, and at least $50 \%$ of patients undergoing weight loss surgery are women of childbearing age [4].

\section{Conclusion}

Although intussusception in adults is rare condition, it is rarer in pregnant patients after bariatric surgery. The incidence might increase with the rise of bariatric surgery. Diagnosis requires a high index of suspicion and an expeditious workup that in- 
cludes appropriate imaging studies. CT scan is the method of choice to confirm the diagnosis. In the case of an inconclusive CT scan and persistent abdominal symptoms, it is essential to proceed to the operating room for exploration without undue delay. There is no current consensus on the optimal treatment, but the literature shows a significantly higher recurrence rate of $33.3 \%$ for only reduction $+/$ - plication in comparison to $7.7 \%$ for resection with reconstruction of the JJ. For this reason, we advise the resection. Further study is needed to better elucidate the mechanism of adult intussusception after RYGB so that the surgical techniques may be improved in an attempt to prevent or minimize the occurrence of this potentially fatal complication.

\section{Conflicts of Interest}

None.

\section{Funding}

None.

\section{References}

1. Samuel I, Mason EE, Renquist KE, Huang YH, Zimmerman MB, Jamal M. Bariatric surgery trends: an 18-year report from the International Bariatric Surgery Registry. Am J Surg. 2006;192(5):657-662.

2. Koppman JS, Li C, Gandsas A. Small bowel obstruction after laparoscopic Roux-en-Y gastric bypass: a review of 9,527 patients. J Am Coll Surg. 2008;206(3):571-584.

3. Edwards MA, Grinbaum R, Ellsmere J, Jones DB, Schneider BE. Intussusception after Roux-en-Y gastric bypass for morbid obesity: case report and literature review of rare complication. Surg Obes Relat Dis. 2006;2(4):483489.

4. Wax JR, Pinette MG, Cartin A, Blackstone J. Female reproductive issues following bariatric surgery. Obstet Gynecol Surv. 2007;62(9):595-604.

5. Torres-Villalobos GM, Kellogg TA, Leslie DB, Antanavicius G, Andrade RS, Slusarek B, Prosen TL, et al. Small bowel obstruction and internal hernias during pregnancy after gastric bypass surgery. Obes Surg. 2009;19(7):944950.

6. Wax JR, Wolff R, Cobean R, Pinette MG, Blackstone J, Cartin A. Intussusception complicating pregnancy following laparoscopic Roux-en-Y gastric bypass. Obes Surg. 2007;17(7):977-979.

7. Azar T, Berger DL. Adult intussusception. Ann Surg. 1997;226(2):134-138.

8. Zubaidi A, Al-Saif F, Silverman R. Adult intussusception: a retrospective review. Dis Colon Rectum. 2006;49(10):1546-1551.

9. Begos DG, Sandor A, Modlin IM. The diagnosis and management of adult intussusception. Am J Surg. 1997; 173(2):88-94.

10. Barussaud M, Regenet N, Briennon X, de Kerviler B, Pessaux P, Kohneh-Sharhi N, Lehur PA, et al. Clinical spectrum and surgical approach of adult intussusceptions: a multicentric study. Int J Colorectal Dis. 2006;21(8):834839. 